\title{
Measurement of Cerebral Venous Oxyhemoglobin Saturation in Children by Near-Infrared Spectroscopy and Partial Jugular Venous Occlusion
}

\author{
C. W. YOXALL, A. M. WEINDLING, N. H. DAWANI, AND I. PEART \\ Department of Child Health, University of Liverpool [C.W.Y, A.M.W.], Liverpool Maternity Hospital \\ [N.H.D.], and Royal Liverpool Children's Hospital, Alder Hey [1.P.], Liverpool, United Kingdom
}

\begin{abstract}
Changes in cerebral venous oxyhemoglobin saturation reflect changes in the balance between cerebral oxygen delivery and cerebral oxygen consumption. Invasive monitoring of cerebral venous saturation $\left(\mathrm{CSvO}_{2}\right)$ has provided useful information in the management of critically ill adults at risk of cerebral hypoxia. This study describes the development and validation of a noninvasive method of measuring $\mathrm{CSvO}_{2}$ suitable for use in sick neonates using near-infrared spectroscopy (NIRS) and partial jugular venous occlusion. This technique was validated by comparison with an invasive measurement of $\mathrm{CSvO}_{2}$, co-oximetry of jugular bulb blood obtained during cardiac catheterization. Agreement between the two methods was assessed using the method of J. M. Bland and D. G. Altman. Fifteen children were studied, aged 3 mo to $14 \mathrm{y}$ (median $2 \mathrm{y}$ ). $\mathrm{CSvO}_{2}$ by co-oximetry ranged from 36 to $80 \%$ (median $60 \%$ ). The mean difference (Co-Oximeter - NIRS) was $1.5 \%$. Limits of agreement were
\end{abstract}

ABSTRACT
The key to successful neonatal intensive care is the provision of sufficient oxygen to tissues. Information about oxygen delivery to, and oxygen consumption by, those tissues is essential to guide therapies aimed at optimizing tissue oxygenation.

The calculation of oxygen delivery and consumption in any vascular bed requires measurements of blood flow, hemoglobin concentration, arterial oxyhemoglobin saturation, and venous oxyhemoglobin saturation and is determined by the two following equations.

$$
\text { Oxygen delivery }=\text { blood flow } \times \mathrm{CaO}_{2}
$$

Oxygen consumption $=$ blood flow $\times\left(\mathrm{CaO}_{2}-\mathrm{CvO}_{2}\right)$

where $\mathrm{CaO}_{2}=$ arterial oxygen content and $\mathrm{CvO}_{2}=$ venous oxygen content.

Received September 15, 1994; accepted March 16, 1995.

Correspondence: Dr. C. W. Yoxall, Clinical Research Fellow, Neonatal Intensive Care Unit, Liverpool Maternity Hospital, Oxford Sireet, Liverpool L7 7BN, UK.

Supported by the Newborn Appeal. C.W.Y. is supported by a Children Nationwide Training Fellowship.
-12.8 to $15.9 \%$. Three different methods of analyzing the NIRS signal were compared. The best agreement was obtained when the changes occurring during the first $5 \mathrm{~s}$ of partial jugular venous occlusion were studied. Greatest accuracy was seen in those subjects with least movement artifact, and we believe this technique will be reliable in sick neonates. (Pediatr Res 38: 319-323, 1995)

\section{Abbreviations}

$\mathrm{CSvo}_{2}$, cerebral venous saturation

$\mathrm{Hbo}_{2}$, oxyhemoglobin

$\mathbf{H b}$, deoxyhemoglobin

NIRS, near-infrared spectroscopy

$\mathrm{CaO}_{2}$, arterial oxygen content

$\mathrm{Cro}_{2}$, venous oxygen content

$\mathrm{So}_{2}$, oxyhemoglobin saturation

$\mathrm{CaO}_{2}$ and $\mathrm{CvO}_{2}$ may in turn be calculated from:

Blood oxygen content $=\left([\mathrm{Hb}] \times \mathrm{So}_{2} \times 1.39\right)+\left(\mathrm{Po}_{2} \times 0.003\right)$ where $[\mathrm{Hb}]=$ hemoglobin concentration, $\mathrm{So}_{2}=$ oxyhemoglobin saturation, $1.39=$ the stoichiometric oxygen capacity of hemoglobin $(\mathrm{mL} / \mathrm{g})$, and $0.003=$ the Bunsen solubility coefficient for oxygen.

From this it can be seen that a decrease in oxygen delivery relative to oxygen consumption will lead to a fall in venous oxyhemoglobin saturation. Similarly, a relative rise in oxygen delivery will lead to a rise in venous oxyhemoglobin saturation. Thus, $\mathrm{CSvO}_{2}$ measurements may be expected to provide useful information about cerebral oxygen dynamics and responses to clinical interventions among patients at risk of cerebral hypoxia. As the consequences of cerebral hypoxia and ischemia are the major cause of long term morbidity in survivors of neonatal intensive care, a measurement of $\mathrm{CSvO}_{2}$ might be expected to provide information of great importance to the understanding of pathophysiology in this group and possibly to help in guiding therapy. 
$\mathrm{CSvO}_{2}$ has been measured by measurement of jugular bulb saturation in adults undergoing cardiopulmonary bypass, neuroanesthesia, and after serious head injury (1-3). These measurements have been used successfully to guide therapy. However, measurement of jugular bulb saturation requires retrograde catheterization of the internal jugular vein and either co-oximetry of intermittent samples, or continuous oximetry with a fiberoptic catheter. Such techniques are not technically feasible in sick infants but are possible in the cardiac catheter laboratory.

The technique of NIRS of tissues offers the possibility of providing hemodynamic data noninvasively. It relies on the fact that tissues scatter, rather than absorb, near-infrared light. The major biologic compounds which absorb this light are $\mathrm{Hbo}_{2}, \mathrm{Hb}$, and oxidized cytochrome $a a_{3}$. Changes in the absorbance of the near-infrared light transmitted through tissues can, therefore, be converted into changes in the concentration of these three substances.

NIRS does not differentiate between hemoglobin in the different vascular compartments. Measurement of $\mathrm{CSvO}_{2}$ requires a constant tissue hemoglobin concentration, indicating a stable flow of blood into and out of the brain. If venous blood volume only is then increased, $\mathrm{CSvO}_{2}$ may be measured from:

$$
\mathrm{CSVO}_{2}=\frac{\Delta\left[\mathrm{Hbo}_{2}\right]}{\Delta[\mathrm{Hbt}]}
$$

where $[\mathrm{Hbt}]=\left[\mathrm{HbO}_{2}\right]+[\mathrm{Hb}]$.

Measurement of $\mathrm{CSvO}_{2}$ by NIRS in human neonates has been reported $(4,5)$. Both of these studies used a head down tilt to increase cerebral venous blood volume. In neither of these studies were measurements validated by an invasive measurement and one study successfully induced an adequate increase in cerebral blood volume in only 19 of 32 subjects (5).

We propose that a more reliable method for increasing cerebral venous blood volume for the measurement of $\mathrm{CSvO}_{2}$ may be partial jugular venous occlusion. This report describes the use of this technique and its validation by comparison with co-oximetry of blood from the jugular bulb of patients undergoing cardiac catheterization.

\section{METHODS}

Patients. All subjects were patients having a cardiac catheter study via a femoral venous approach. Catheterization was performed either under general anesthetic or local anesthetic and sedation. Fifteen subjects were studied. Median (range) age of the subjects was 2 y ( 3 mo to $14 \mathrm{y}$ ). Three children were having catheter occlusion of a patent ductus arteriosus, four were having assessment of a ventricular septal defect, three were having studies after previous corrective surgery, one had primary pulmonary hypertension, and four were having assessment of complex cyanotic abnormalities.

\section{NIRS}

Cerebral NIRS was performed using a Niro 500 (Hamamatsu Photonics, Japan). Optodes were positioned $4 \mathrm{~cm}$ apart on an area of non-hair-bearing scalp, usually near the right fronto-temporal hairline, and fixed in place with doublesided adhesive rings. NIRS measurements were recorded every $0.5 \mathrm{~s}$.

Partial jugular venous occlusion. Before the start of the cardiac catheter study several partial jugular venous occlusions were performed. These were caused by gentle pressure applied to one side of the patient's neck for 5 to $10 \mathrm{~s}$ only. This period was chosen to provide an adequate number of NIRS data points with minimal hemodynamic disturbance of the subject. A unilateral occlusion was used initially. If this did not cause a detectable rise in cerebral blood volume then bilateral occlusion was used.

Analysis of NIRS signal. The changes in absorbance of each of the four wavelengths used were saved into a computer file using software supplied by Hamamatsu Photonics UK. These values were converted into changes in the concentration of $\mathrm{HbO}_{2}$ and $\mathrm{Hb}$ in $\mathrm{mM} \cdot \mathrm{cm}$ using the known absorption spectra of these chromophores (6).

The changes in $\mathrm{Hbo}_{2}$ and $\mathrm{Hbt}$ concentration were plotted against time for each episode of partial jugular venous occlusion. A typical occlusion is illustrated in Fig 1. The data were visually inspected, and an occlusion was determined as satisfactory for further analysis if there was a steady baseline before the occlusion, a rise in both $\mathrm{Hbo}_{2}$ and $\mathrm{Hb}$ during the occlusion and a return to the same baseline after release of the occlusion.

The baseline of $\Delta \mathrm{HbO}_{2}$ and $\Delta \mathrm{Hbt}$ for each occlusion was calculated by averaging the ten data points during $5 \mathrm{~s}$ immediately before the rise in $\mathrm{HbO}_{2}$ and $\mathrm{Hb} . \Delta \mathrm{HbO}_{2}$ and $\Delta \mathrm{Hbt}$ were calculated in three different ways. 1) Area under the curves. This method calculated $\mathrm{SvO}_{2}$ from the area under the $\mathrm{Hbo}_{2}$ curve expressed as a percentage of the area under the $\mathrm{Hbt}$ curve. 2) Initial change. This method calculated $\mathrm{SvO}_{2}$ at each of the 10 points point during the first $5 \mathrm{~s}$ of venous occlusion as the rise in $\mathrm{HbO}_{2}$ expressed as a percentage of the rise in $\mathrm{Hbt}$. The mean of these ten measurements was then taken. 3) Peak. This method calculated $\mathrm{SvO}_{2}$ as above at each point at the peak of the rise. A mean of these points was taken. If the peak lasted for greater than five seconds, only the first ten points were examined.

Jugular bulb co-oximetry. The catheter was introduced via a femoral vein and was advanced into the jugular bulb under

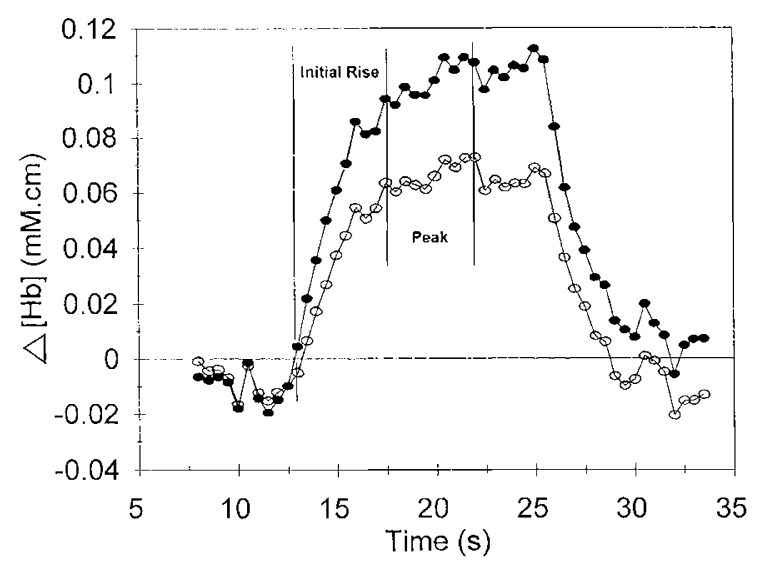

Figure 1. NIRS data obtained during partial jugular venous occlusion and methods used to calculate $\mathrm{CSvO}_{2} . \mathrm{O},\left[\mathrm{Hbo}_{2}\right] ; \boldsymbol{\bullet},[\mathrm{Hbt}]$. 
$x$-ray screening. During the period when the partial jugular venous occlusions were being performed two samples of blood were drawn from the jugular bulb. Oxygen saturation was measured by co-oximetry (OSM2, Radiometer, Copenhagen, Denmark or Oxicom, Waters Instruments, Rochester, MN).

Comparison of techniques. The median of the co-oximeter measurements for each subject was compared with the median of the NIRS measurements using the standard technique for assessing agreement between two methods of clinical measurement as described by Bland and Altman (7).

This study had the approval of the hospital ethics committee. Informed parental consent was obtained for each subject.

\section{RESULTS}

The median (range) study duration was 6.3 (2.8 to 18.5 ) minutes. The results are summarized in Table 1. Partial jugular venous occlusion caused a satisfactory rise in cerebral blood volume in all subjects. Not all occlusions were successful, particularly in those subjects who had not received a general anesthetic. The time available for occlusions to be performed was dictated by clinical considerations. The number of successful occlusions obtained varied between subjects (median 4, range 1-16).

The median (range) $\mathrm{CSvO}_{2}$ by co-oximeter was $60 \%$ (36$80 \%$ ). Median (range) $\mathrm{CSvO}_{2}$ measured by NIRS was $60 \%$ (43-77\%) when the area under the curve was used, $56 \%$ $(40-82 \%)$ when the initial change was analyzed, and $61 \%$ (43-77\%) when the peak of the curve was analyzed.

Analysis of the agreement between co-oximetry and NIRS using the Bland and Altman technique is shown in Table 2. The mean difference between the co-oximeter and the NIRS values was $0.9 \%$ when the area under the curves or the peak of the rise was analyzed and $1.5 \%$ when the initial change was analyzed. The limits of agreement were narrowest when the initial change was analyzed $(-12.8$ to $15.9 \%)$. There was close
Table 2. Agreement between co-oximetry and NIRS

\begin{tabular}{lccc} 
& Area & Initial Rise & Peak \\
\hline Mean difference (\%) & 0.9 & 1.5 & 0.9 \\
95\% CI & -2.5 to 5.6 & -2.5 to 5.6 & -3.5 to 5.3 \\
Limits of agreement $(\%)$ & -18.8 to 20.5 & -12.8 to 15.9 & -14.6 to 16.3 \\
$95 \%$ Cl & & & \\
Lower limit & -9.2 to -28.5 & -5.8 to -19.9 & -7.0 to -22.1 \\
Upper limit & 10.9 to 30.2 & 8.9 to 22.9 & 8.8 to 23.9 \\
\hline
\end{tabular}

$\mathrm{Cl}$, confidence interval.

agreement between the two methods of measurement across a wide range of $\mathrm{CSvO}_{2}$ values, as illustrated (Fig. 2).

Cardiac catheter studies were performed under general anesthetic or sedation and local anesthetic. We found the NIRS studies with the closest agreement were obtained from subjects having a general anesthetic. This was attributed to less movement artifact. We also noted that there was less noise in the NIRS signal obtained from smaller subjects. A larger number of satisfactory curves were obtained during the shortest time in these individuals and these tended to show better agreement. For the 10 subjects in whom three or more successful NIRS measurements were made, the co-oximetry value was always included within the median NIRS value \pm 1.5 times the interquartile range. In the five subjects with fewer than three NIRS measurements, the co-oximetry value was included within the median NIRS value \pm 1.5 times the interquartile range in one subject.

The data from the study from which the most uniform NIRS curves were obtained (subject 5) are illustrated (Fig. 3). $\mathrm{CSvO}_{2}$ by NIRS was $62 \%(95 \% \mathrm{CI}=59$ to $64 \%)$. The coefficient of variation for these 16 NIRS measurement was $8 \% . \mathrm{CSvO}_{2}$ by co-oximetry in this subject was $64 \%$.

There were two co-oximetry measurements in 13 subjects. $\mathrm{CSvO}_{2}$ measured by co-oximetry usually varied by less than $5 \%$ during the study. However, on one occasion (subject 6 ) the

Table 1. Results for individual subjects

\begin{tabular}{|c|c|c|c|c|c|c|c|c|c|c|c|}
\hline \multirow[b]{2}{*}{ Subject } & \multicolumn{3}{|c|}{ Co-oximeter } & \multicolumn{2}{|c|}{ NIRS } & \multicolumn{2}{|c|}{ Area } & \multicolumn{2}{|c|}{ Initial rise } & \multicolumn{2}{|c|}{ Peak } \\
\hline & Samples & $\begin{array}{c}\text { Median } \\
(\%)\end{array}$ & $\pm 1.5 \times \mathrm{IQR}$ & Occ. & Meas. & $\begin{array}{c}\text { Median } \\
(\%)\end{array}$ & $\pm 1.5 \times \mathrm{IQR}$ & $\begin{array}{c}\text { Median } \\
(\%)\end{array}$ & $\pm 1.5 \times$ IQR & $\begin{array}{c}\text { Median } \\
(\%)\end{array}$ & $\pm 1.5 \times \mathrm{IQR}$ \\
\hline 1 & 2 & 67 & $58-76$ & 5 & 2 & 51 & $48.8-52.7$ & 56 & $55.9-56.8$ & 52 & $48.8-55.4$ \\
\hline 2 & 2 & 60 & $57.3-61.8$ & 10 & 2 & 47 & $39.3-53.8$ & 52 & $50.8-53.7$ & 46 & $42.7-50$ \\
\hline 3 & 2 & 67 & $65.8-67.3$ & 7 & 5 & 64 & $55.5-72.5$ & 65 & $59.6-70.8$ & 64 & $56-72.5$ \\
\hline 4 & 2 & 68 & $62.3-72.8$ & 15 & 4 & 70 & $55.3-84.7$ & 65 & $45.3-84.9$ & 71 & $55.3-86.2$ \\
\hline 5 & 2 & 64 & $62.5-65.5$ & 16 & 16 & 62 & $48.3-75.7$ & 62 & $53.1-71$ & 61 & $50.3-71.5$ \\
\hline 6 & 2 & 75 & $55.5-94.5$ & 13 & 4 & 69 & $40.6-96.4$ & 82 & $56.8-107.6$ & 77 & $29.9-124.5$ \\
\hline 7 & 1 & 36 & & 11 & 3 & 54 & $26.9-80.1$ & 40 & $11.9-68.3$ & 49 & $29.9-67.4$ \\
\hline 8 & 2 & 59 & $57.8-59.3$ & 8 & 5 & 60 & $35.3-84.3$ & 51 & $42.2-59$ & 61 & $35.8-85.2$ \\
\hline 9 & 1 & 55 & & 12 & 6 & 64 & $48.3-79$ & 66 & $50.4-82.1$ & 64 & $49.8-79$ \\
\hline 10 & 1 & 80 & & 2 & 1 & 77 & & 77 & & 77 & \\
\hline 11 & 2 & 37 & $34.3-38.8$ & 5 & 2 & 56 & $44.1-68.1$ & 49 & $20.9-76.1$ & 46 & $31.7-59.3$ \\
\hline 12 & 2 & 70 & $67.3-71.8$ & 19 & 7 & 64 & $36.8-90.8$ & 61 & $33.7-88.6$ & 66 & $40.7-90.8$ \\
\hline 13 & 2 & 53 & $51.8-53.3$ & 3 & 3 & 43 & $32.9-53.3$ & 50 & $31.2-68.1$ & 43 & $27.8-59.3$ \\
\hline 14 & 2 & 58 & $55.3-59.8$ & 3 & 2 & 52 & $45.3-59$ & 47 & $39.8-53.7$ & 54 & $42-64.9$ \\
\hline 15 & 2 & 53 & $51.7-53.8$ & 14 & 5 & 54 & $41.3-67.5$ & 52 & $39.1-65.3$ & 54 & $43.8-65.1$ \\
\hline $\begin{array}{l}\text { Median } \\
\text { (Range) }\end{array}$ & 2 & $\begin{array}{r}60 \\
(36-80)\end{array}$ & & $\begin{array}{r}10 \\
(2-19)\end{array}$ & $\begin{array}{r}4 \\
(1-16)\end{array}$ & $\begin{array}{r}60 \\
(43-77)\end{array}$ & & $\begin{array}{r}56 \\
(40-82)\end{array}$ & & $\begin{array}{r}61 \\
(43-77)\end{array}$ & \\
\hline
\end{tabular}

Occ., number of occlusions performed, meas., number of successful measurements made, and IQR, interquartile range. 


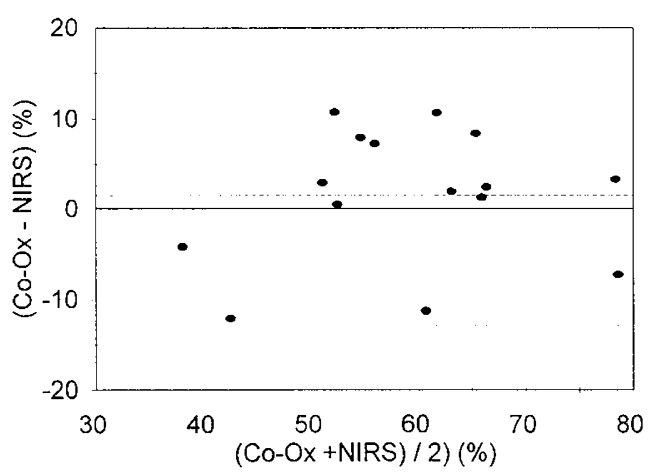

Figure 2. The difference between $\mathrm{CSVO}_{2}$ measurement by co-oximetry and NIRS (initial rise) for each subject plotted against the mean of each pair of measurements. - - - , mean difference $=1.5 \%$; $\cdots .$. , limits of agreement $=$ -12.8 to 15.9 .

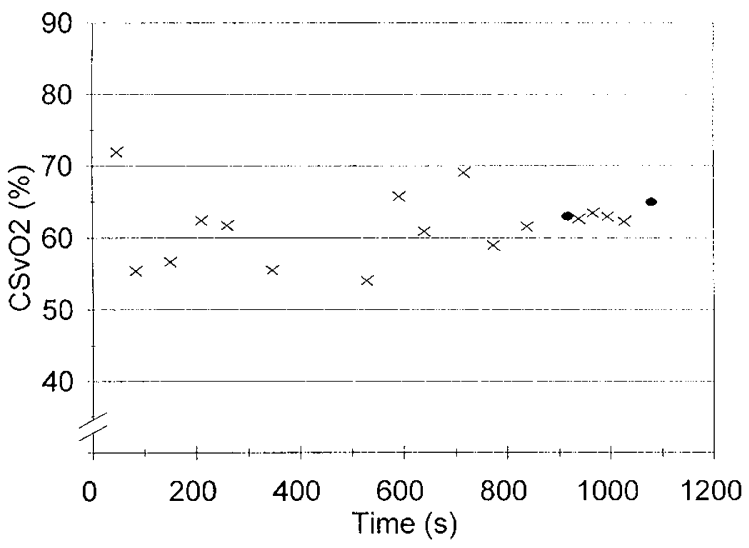

Figure 3. $\mathrm{CSvO}_{2}$ measurements $v s$ time for subject $5 . \times$, co-oximeter measurement; 9 , NIRS measurement.

two co-oximetry values were 88 and $62 \%$, but the NIRS values were similar ( 88 and $66 \%$ ). In only three of these 13 cases was the median NIRS value included in the median co-oximetry value \pm 1.5 times the interquartile range.

\section{DISCUSSION}

NIRS with partial jugular venous occlusion allows estimation of $\mathrm{CSvO}_{2}$. We investigated three different methods of analyzing the NIRS data to determine which period during venous occlusion provided the best information. Although there was little difference between the three methods used, the limits of agreement suggested that the most accurate estimate appeared to be made when the initial rise in cerebral blood volume was analyzed. We have considered the possible reason for this and have concluded that it may be that the blood involved in the initial rise is representative of the situation immediately before the partial jugular venous occlusion. Venous occlusion may affect tissue oxygenation by eventually impairing arterial flow, and this would lead to an increased oxygen extraction. We have noted during prolonged venous occlusion in the adult forearm that $\mathrm{Hb}$ continued to rise whereas $\mathrm{Hbo}_{2}$ eventually started to fall.

In the course of this study it became apparent that it was important to analyze only events where there was a steady baseline for cerebral $\mathrm{HbO}_{2}$ and $\mathrm{Hb}$ concentrations before and after the occlusion. Instability of the baseline was felt to be due to subject movement resulting in interrogation of a different area of cerebrum or, possibly, alterations in cerebral hemodynamics.

Although the agreement between the two methods appears to be acceptable, it is possible that the study design led to it being underestimated. We took the median of the measurements made by both techniques on an individual subject during the study period as the $\mathrm{CSvO}_{2}$ by that method for that subject. However, studies varied in duration and in the number and temporal relationship of co-oximetry and NIRS measurements. This led to two potential sources of error. Firstly changes in the "true" $\mathrm{CSvO}_{2}$ during a study and clustering of measurements made by the two different techniques at different times may have resulted in some of the differences between the two measurements. However, as $\mathrm{CSvO}_{2}$ by co-oximetry was fairly stable in most subjects, this was likely to have exerted only a small effect. Secondly, the repeatability of each method was considered to determine whether that might account for any of the differences between the two methods. When a set of measurements contains a small number of data points, repeatability of the measurement may be expressed as 1.5 times the interquartile range about the median observation. Since the median co-oximeter value was included in this range in 11 of the 15 subjects it would appear that at least some of the difference observed may have been due to variability in the NIRS measurement. For the co-oximetry measurements 1.5 times the interquartile range was usually small and probably did not account for any of the difference.

Unilateral jugular venous occlusion appeared to induce a satisfactory rise in cerebral blood volume in most individuals, but some subjects needed bilateral occlusion. This may reflect the proportion of cerebral venous blood draining via the right jugular vein, or the adequacy of intracranial venous anastomoses in those individuals.

Application of this method to infants undergoing neonatal intensive care may provide useful information. We have found that it is an easy measurement to make in this setting, with no apparent distress caused to the babies and no observed change in heart rate or arterial saturation as measured by pulse oximetry. There may be concern that this measurement adversely affects cerebral hemodynamics and might possibly predispose to intracranial hemorrhage. We think that this is highly unlikely. The measurement period is very short and the estimated increase in cerebral blood volume is in the order of 0.25 to 0.33 $\mathrm{mL} / 100 \mathrm{~mL}$ using a path length correction factor of $3.85(8)$. We have observed much larger increases in cerebral blood volume lasting for longer periods occurring in ventilated infants who have actively expired against the ventilator and during a Valsalva maneuver by nonventilated infants. As abolition of these effects by muscular paralysis has not been shown to reduce the incidence of intracranial hemorrhage, it seems improbable that the increase in cerebral blood volume caused by partial, unilateral jugular venous occlusion will be harmful.

We conclude that NIRS with partial jugular venous occlusion provides a reasonably reliable estimate of $\mathrm{CSvO}_{2}$ and may be suitable for use in the neonatal intensive care unit. 


\section{REFERENCES}

1. Sheinberg M, Kanter MJ, Robertson CS, Contant CF, Narayan RK, Grossman RG 1992 Continuous monitoring of jugular venous oxygen saturation in head injured patients. J Neurosurg 76:212-217

2. Nakajima T, Kuro M, Hayashi Y, Kitaguchi K, Uchida O, Takaki O 1992 Clinical evaluation of cerebral oxygen balance during cardiopulmonary bypass: On-line continuous monitoring of jugular venous oxyhaemoglobin saturation. Anesth Analg 74:630-635

3. Kawaguchi M, Ohsumi H, Nakajima T, Kuro M 1993 Intra-operative monitoring of cerebral haemodynamics in a patient with Takayashu's arteritis. Anaesthesia 48:496-498

4. Wyatt JS, Cope M, Delpy DT, Wray Susan, Reynolds EOR 1986 Quantification of cerebral oxygenation and haemodynamics in sick newborn infants by near infrared spectrophotometry. Lancet 8515:1063-1066
5. Skov L, Pryds O, Greisen G, Lou H 1993 Estimation of cerebral venous saturation in newborn infants by near infrared spectroscopy. Pediatr Res 33:52-55

6. Wray S, Cope M, Delpy DT, Wyatt JS, Reynolds EOR 1988 Characterisation of the near infrared absorption spectra of cytochrome $a a_{3}$ and haemoglobin for the non-invasive monitoring of cerebral oxygenation. Biochim Biophys Acta 933:184-192

7. Bland JM, Altman DG 1986 Statistical methods for assessing agrcement between two methods of clinical measurement. Lancet 1:307-310

8. Van der Zee P, Cope M, Arridge SR, Essenprejs M, Potter LA, Edwards AD Wyatt JS, McCormick DC, Roth SC, Reynolds EOR, et al. 1992 Experimentally measured optical pathlengths for the adult head, calf and forearm and the head of the newborn infant as a function of interoptode spacing. Adv Exp Med Biol 316:143-153 